\title{
Influence of the Domain Boundary on the Speeds of Traveling Waves
}

\author{
Lanxiang Ma1, Jiale Tan ${ }^{2}$ \\ ${ }^{1}$ Ningbo Shentong Energy Co., Ltd., Ningbo, China \\ ${ }^{2}$ Department of Mathematics, Tongji University, Shanghai, China \\ Email: lanxiangma@163.com, jialetan5293@gmail.com
}

Received 30 April 2014; revised 5 June 2014; accepted 15 June 2014

Copyright (C) 2014 by authors and Scientific Research Publishing Inc.

This work is licensed under the Creative Commons Attribution International License (CC BY).

http://creativecommons.org/licenses/by/4.0/

(c) (i) Open Access

\section{Abstract}

Let $H>0$ be a constant, $g \geq 0$ be a periodic function and $\Omega=\{(x, y)|| x \mid<H+g(y), y \in \mathbb{R}\}$. We consider a curvature flow equation $V=\kappa+A$ in $\Omega$, where for a simple curve $\gamma_{t} \subset \Omega, V$ denotes its normal velocity, $\boldsymbol{\kappa}$ denotes its curvature and $A>0$ is a constant. [1] proved that this equation has a periodic traveling wave $U$, and that the average speed $c$ of $U$ is increasing in $A$ and $H$, decreasing in max $g^{\prime}$ when the scale of $g$ is sufficiently small. In this paper we study the dependence of $c$ on $A, H$, max $g^{\prime}$ and on the period of $g$ when the scale of $g$ is large. We show that similar results as [1] hold in certain weak sense.

\section{Keywords}

\section{Curvature Flow Equation, Traveling Wave, Average Speed, Spatial Heterogeneity}

\section{Introduction}

We study traveling waves for a curvature-driven motion of plane curves in a band domain $\Omega$. The law of motion of the curve is given by

$$
V=\kappa+A \text { on } \gamma_{t} \subset \Omega,
$$

where $\gamma_{t} \subset \Omega$ is a simple, smooth curve, $V$ denotes its normal velocity, $\kappa$ denotes its curvature and $A$ is a positive constant representing a driving force. The band domain $\Omega$ is defined as the following. Set

$$
\mathcal{G}:=\left\{g(y) \mid g(y) \in C^{3}(\mathbb{R}), g(y) \geq g(0)=0, g \text { is 1-periodic, } \max _{y}\left|g^{\prime}(y)\right|<1\right\} .
$$


For some $g \in \mathcal{G}$ we define

$$
\begin{gathered}
\Omega=\Omega(H, g, p) \\
\Omega=\Omega(H, g, p):=\left\{(x, y) \in \mathbb{R}^{2}|| x \mid<H+g_{p}(y), y \in \mathbb{R}\right\},
\end{gathered}
$$

where $H>0$ is a constant and $g_{p}(y):=p g(y / p)$ for some $p>0$ (see Figure 1). Denote the left (resp. right) boundary of $\Omega$ by $\partial_{-} \Omega$ (resp. $\partial_{+} \Omega$ ).

By a solution of (1) we mean a time-dependent simple, smooth curve $\gamma_{t}$ in $\Omega$ which satisfies (1) and contacts $\partial_{ \pm} \Omega$ perpendicularly. Equation (1) appears as a certain singular limit of an Allen-Cahn type nonlinear diffusion equation under the Neumann boundary conditions. The curve $\gamma_{t}$ represents the interface between two different phases (see, e.g., [1]-[4] for details). In physics, chemistry and many other fields, an interface may propagate in a domain with obstacles, say, with obstacles lying in several lines. The motion of the interface between two adjacent lines is then like the propagation of $\gamma_{t}$ in $\Omega$ in our problem. Hence the undulation of the boundary of $\Omega$ can be regarded as effect of obstacles and so it can be in any size. [1] studied the homogenization limit of this problem (as $p \rightarrow 0$ ), we will consider the case where $p$ is large.

To avoid sign confusion, the normal to the curve $\gamma_{t}$ will always be chosen toward the upper region, and the sign of the normal velocity $V$ and the curvature $\kappa$ will be understood in accordance with this choice of the normal direction. Consequently, $\kappa$ is negative at those points where the curve is concave while it is positive where the curve is convex (see Figure 1).

In the case where $\gamma_{t}$ is expressed as a graph of a function $y=u(x, t)$ at each time $t$. Let $\zeta_{-}(t), \zeta_{+}(t)$ be the $x$-coordinates of the end points of $\gamma_{t}$ lying on $\partial_{-} \Omega, \partial_{+} \Omega$, respectively. In other words, $\left(\zeta_{ \pm}(t), u\left(\zeta_{ \pm}(t), t\right)\right) \in \partial_{ \pm} \Omega$. Now (1) is equivalent to

$$
u_{t}=\frac{u_{x x}}{1+u_{x}^{2}}+A \sqrt{1+u_{x}^{2}}, \quad \zeta_{-}(t)<x<\zeta_{+}(t), t>0
$$

with the boundary conditions

$$
u_{x}\left(\zeta_{ \pm}(t), t\right)=\mp g_{p}^{\prime}\left(u\left(\zeta_{ \pm}(t), t\right)\right),
$$

with $\left(\zeta_{ \pm}(t), u\left(\zeta_{ \pm}(t), t\right)\right) \in \partial_{ \pm} \Omega$. The condition $\max _{y}\left|g^{\prime}(y)\right|=\max _{y}\left|g_{p}^{\prime}(y)\right|<1$ in the definition $\mathcal{G}$ prevent $\gamma_{t}$ from developing singularities near the boundary $\partial_{ \pm} \Omega$ (cf. [1]). Denote

$$
\tan \alpha_{g}:=\max _{y} g^{\prime}(y)
$$

and call $\alpha_{g}$ the maximum opening angle of $\Omega(H, g, p)$, or, of $g$. Then $\alpha_{g} \in\left(0, \frac{\pi}{4}\right)$ for $g \in \mathcal{G}$.

Definition 1 A solution $U\left(x, t ; A, H, g, p, \alpha_{g}\right) \in C^{2+\mu, 1+\mu / 2}\left(\left[\zeta_{-}(t), \zeta_{+}(t)\right] \times \mathbb{R}\right)$ for some $\mu \in(0,1)$ (also write as $U(x, t)$ for simplicity) of (3)-(4) is called a periodic traveling wave if it satisfies $U\left(x, t+T_{p}\right)=U(x, t)+p$ for some $T_{p}>0$. Its average speed is defined by

$$
c=c\left(A, H, g, p, \alpha_{g}\right):=\frac{p}{T_{p}} .
$$
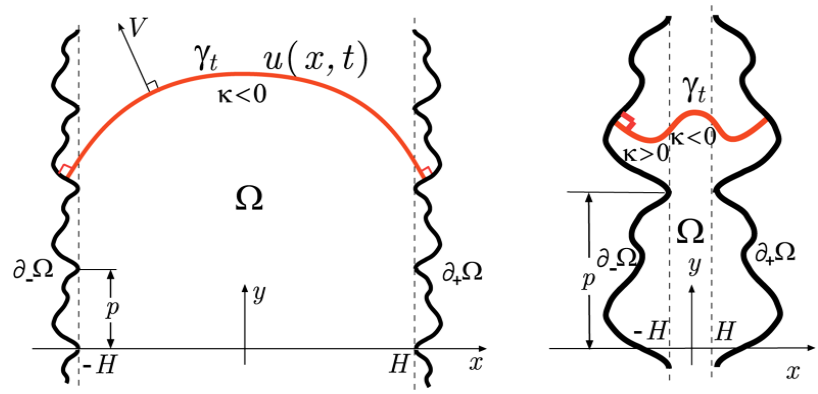

Figure 1. Domain $\Omega$ (the left one has fine boundaries, the right one has coarse boundaries). 
In [1] the authors proved that, under the condition $A H>\sin \alpha_{g}$, the problem (3)-(4) has a periodic traveling wave $U(x, t)$, it is unique under the normalization condition $U(0,0)=0$ and

$$
\left\{\begin{array}{l}
U(x, t)=U(-x, t), \quad U_{t}(x, t)>0, \\
\min _{y} g^{\prime}(y) \leq-\operatorname{sgn} x \cdot U_{x}(x, t) \leq \tan \alpha_{g},
\end{array}\right.
$$

for all $t \in \mathbb{R}$ and $x$ where $U$ is defined. In addition, [1] studied the homogenization limit of the average speed $c$.

Theorem A (Theorem 2.3 in [1]). Assume that $A H>\sin \alpha_{g}$. Let $U(x, t)$ be the periodic traveling wave of (3)-(4) with average speed $c=c\left(A, H, g, p, \alpha_{g}\right)$. Then

$$
\bar{c}<c<\bar{c}+M \sqrt{p} \text { as } p \rightarrow 0,
$$

where $\bar{c}=\bar{c}\left(A, H, \alpha_{g}\right)$ is the constant determined uniquely by

$$
H=\int_{0}^{\alpha_{g}} \frac{\cos r \mathrm{~d} r}{A-\bar{c} \cos r}
$$

and $M$ is a positive constant independent of $p$. Moreover $\bar{C}$ satisfies

$$
0<\bar{c}<A, \quad \frac{\partial \bar{c}}{\partial \alpha_{g}}<0, \quad \frac{\partial \bar{c}}{\partial A}>0, \quad \frac{\partial \bar{c}}{\partial H}>0
$$

Theorem A gives the dependence of $c$ on $A, H$ and $\alpha_{g}$ near the homogenization limit (as $p \rightarrow 0$ ). It is known that in the study of spatially heterogeneous problems, homogenization is a powerful method when the spatial heterogeneity is fine (for example, $p \rightarrow 0$ in our problem) (cf. [5] [6]). On the contrary, the mathematical analysis is completely different and very difficult when the spatial heterogeneity is coarse (for example, $p>0$ is large in our problem). How does the traveling wave $U$ and its average speed $c$ in our problem depend on the parameters $A, H, g, \alpha_{g}$ and $p$ when $p$ is large? This is an interesting problem in physics and is also a challenging one in mathematics. Some mathematicians from Japan and France have been working on it for several years, but yet very little is known so far. Our main purpose in this paper is to study this problem by analytic and numerical methods, and try to give some answers.

This paper is arranged as the following. In section 2 we list some notations and present our main theorem. In section 3 we prove the main theorem. In subsection 3.1 we prove that $c\left(A, H, g, p, \alpha_{g}\right)$ is increasing in $A$; in subsection 3.2 we prove that $c\left(A, H_{k}, g, p, \alpha_{g}\right)$ is increasing in some increasing sequence $\left\{H_{k}\right\}$; in subsection 3.3 we prove that $c\left(A, H, g_{i}, p, \alpha_{g_{i}}\right)$ is increasing in some decreasing sequence $\left\{\alpha_{g_{i}}\right\}$. Finally, in section 4 we present some numerical simulation results, including the dependence of $c$ on the period $p$ of $g_{p}$.

\section{Notations and Main Results}

We list some notations for convenience. For any $g \in \mathcal{G}, A>0, H>0$ and $p>0$, denote

$$
\begin{gathered}
B(g):=\max _{y} g(y), \quad S(g):=\max _{y}\left|g^{\prime}(y)\right|, \\
J(A, g):=A\left[1+(S(g))^{2}\right]^{3 / 2}, \quad d(A, g, p):=\sqrt{\frac{p}{2 J(A, g)},} \\
Q(A, g, p):=p B(g) J(A, g)+2 \sqrt{2 p J(A, g)}, \\
N:=\max \left\{n \in \mathbb{N} \mid n Q(A, g, p)<\tan \alpha_{g}\right\}, \\
K_{1}:=\min \left\{2 H^{2} J(A, g),\left(\frac{\sqrt{2+B(g) \tan \alpha_{g}}-\sqrt{2}}{\sqrt{J(A, g)} B(g)}\right)^{2}\right\}, \\
K_{2}:=\min \left\{2 A H^{2},\left(\frac{\sqrt{2+B(g) \tan \alpha_{g}}-\sqrt{2}}{\sqrt{J(A, g)} B(g)}\right)^{2}\right\} .
\end{gathered}
$$


Clearly, $N$ depends on $A, g, p, \alpha_{g}$ and $K_{1}, K_{2}$ depend on $A, H, g, \alpha_{g}$. Finally, for any $\alpha \in\left(0, \frac{\pi}{4}\right), B_{0}>0$, $0<S_{0}<1$, denote

$$
\mathcal{G}\left(\alpha, B_{0}, S_{0}\right):=\left\{g \in \mathcal{G} \mid B(g) \leq B_{0}, \alpha_{g}=\alpha, S(g) \leq S_{0}\right\} .
$$

Here is an example, let $g(y)=(1+\sin 2 \pi y) /(3 \pi) \in \mathcal{G}, \quad H=p=1, \quad A=2(9 / 13)^{3 / 2}$, then we have

$$
\begin{gathered}
B(g)=\frac{2}{3 \pi}, \quad S(g)=\frac{2}{3}, \quad J(A, g)=2, \\
d(A, g, p)=\frac{1}{2}, \quad Q(A, g, p)=\frac{4}{3 \pi}+4, \\
K_{1}=K_{2}=\frac{9 \pi^{2}+\pi-3 \pi \sqrt{9 \pi^{2}+2 \pi}}{2} .
\end{gathered}
$$

It is easily seen that

$$
\begin{gathered}
p<K_{1} \Rightarrow d(A, g, p)<H \text { and } Q(A, g, p)<\tan \alpha_{g} . \\
p<K_{2} \Rightarrow\left\{\begin{array}{l}
d(A, g, p)<\sqrt{\frac{p}{2 A}}<H, \\
Q(A, g, p)<\tan \alpha_{g} .
\end{array}\right.
\end{gathered}
$$

Therefore, if $p<K_{1}$ or $p<K_{2}$ holds, then $N\left(A, g, p, \alpha_{g}\right) \geq 1$.

The following is our main result.

Main Theorem. Assume $g \in \mathcal{G}$ and $A H>\sin \alpha_{g}$. Then

1) $c\left(A, H, g, p, \alpha_{g}\right)$ is strictly increasing in $A$;

2) if $p<K_{1}\left(A, H, g, \alpha_{g}\right)$, then $c\left(A, H_{k}, g, p, \alpha_{g}\right)$ is strictly increasing in $k$, where $H_{k}:=H+\left(2^{k}-1\right)[H-d(A, g, p)]$ for $k=0,1,2, \cdots$;

3) if $p<K_{2}\left(A, H, g, \alpha_{g}\right)$, then $c\left(A, H, g_{k}, p, \alpha_{k}\right)$ is strictly increasing in $k \in\{0,1, \cdots, N\}$, where $N$ is given by (11), $g_{k} \in \mathcal{G}\left(\alpha_{k}, B(g), S(g)\right)$ and

$$
\alpha_{k}:=\arctan \left[\tan \alpha_{g}-k Q(A, g, p)\right]
$$

for $k=0,1, \cdots, N$.

We remark that 3) of the theorem mainly states the dependence of $c$ on $\alpha$ but not on $g$ itself. In fact, for $\alpha_{k}$ defined by (14), the conclusion of 3) holds for any $g_{k}$ provided $\max g_{k}^{\prime}=\tan \alpha_{k}$ (with restrictions $B\left(g_{k}\right) \leq$ $\left.B(g), \quad S\left(g_{k}\right) \leq S(g)\right)$, the exact shape of $g_{k}$ does not matter.

By the main theorem, $c\left(A, H, g, p, \alpha_{g}\right)$ is increasing in continuously varying $A$, but it is increasing in $H$ and decreasing in $\alpha_{g}$ only in weak sense, that is, the monotonicity holds only for certain sequences. It turns out that the monotonicity for continuously varying $H$ and $\alpha_{g}$ is very difficult. In fact, we believe that $\partial c / \partial \alpha_{g}<0$ is not true when $p$ is large. This is quite different from the case where $p \rightarrow 0$.

\section{Proof of the Main Theorem}

In this section, for any two solutions $u_{1}$ and $u_{2}$ of (3)-(4), when we write $u_{1} \leq u_{2}$ or $u_{1}<u_{2}$ we mean that the inequality holds on the common domain where $u_{1}$ and $u_{2}$ are defined.

\subsection{Proof of Main Theorem 1}

Assume that $A_{1}>A_{2}>\sin \alpha_{g} / H$. For $i=1,2$, denote $U_{i}(x, t):=U\left(x, t ; A_{i}, H, g, p, \alpha_{g}\right)$ the (unique) periodic traveling wave of (3)-(4) for $A=A_{i}$, denote the $x$-span for each $t$ by $\left[\zeta_{-}^{i}(t), \zeta_{+}^{i}(t)\right]$. Denote the time-period of $U_{i}$ by $T_{i}$, that is,

$$
U_{i}\left(x, t+T_{i}\right)=U_{i}(x, t)+p \text { for all } t \in \mathbb{R} .
$$


Let $\tau_{1}, \tau_{2}$ be two times such that

$$
U_{2}\left(x, \tau_{2}\right) \leq U_{1}\left(x, \tau_{1}\right), \quad U_{2}\left(x, \tau_{2}\right) \neq U_{1}\left(x, \tau_{1}\right) \text { and } U_{2}\left(x_{0}, \tau_{2}\right)=U_{1}\left(x_{0}, \tau_{1}\right)
$$

for some $x_{0}$. This is possible since $U_{1}(x, t) \not \equiv U_{2}(x, t)$. Define

$$
w(x, t):=U_{1}\left(x, t+\tau_{1}\right)-U_{2}\left(x, t+\tau_{2}\right)
$$

for $x \in\left[\xi_{-}(t), \xi_{+}(t)\right], t \geq 0$, where

$$
\begin{gathered}
\xi_{-}:=\max \left\{\zeta_{-}^{1}\left(t+\tau_{1}\right), \zeta_{-}^{2}\left(t+\tau_{2}\right)\right\}, \\
\xi_{+}:=\min \left\{\zeta_{+}^{1}\left(t+\tau_{1}\right), \zeta_{+}^{2}\left(t+\tau_{2}\right)\right\}
\end{gathered}
$$

Then $w(x, t)$ satisfies

$$
\left\{\begin{array}{l}
w_{t}=a_{1}(x, t) w_{x x}+b_{1}(x, t) w_{x}, \quad x \in\left(\xi_{-}(t), \xi_{+}(t)\right), \\
w(x, 0) \geq 0, \quad w(x, 0) \neq 0, \quad w\left(x_{0}, 0\right)=0,
\end{array}\right.
$$

where

$$
\begin{gathered}
a_{1}(x, t)=\frac{1}{1+U_{1 x}^{2}}, \\
b_{1}(x, t)=\frac{A\left(U_{1 x}+U_{2 x}\right)}{\sqrt{1+U_{1 x}^{2}}+\sqrt{1+U_{2 x}^{2}}}-\frac{\left(U_{1 x}+U_{2 x}\right) U_{2 x x}}{\left(1+U_{1 x}^{2}\right)\left(1+U_{2 x}^{2}\right)}
\end{gathered}
$$

are both bounded functions. We show that

$$
w(x, t)>0 \text { for } x \in\left[\xi_{-}(t), \xi_{+}(t)\right], t>0 .
$$

First by the maximum principle (see, for example, Theorem 2 in Chapter 3 in [7]) we have

$$
w(x, t)>0 \text { for } x \in\left(\xi_{-}(t), \xi_{+}(t)\right), t>0 .
$$

This implies that the graph of $U_{2}\left(x, t+\tau_{2}\right)$ can not touch the graph of $U_{1}\left(x, t+\tau_{1}\right)$ from below except on their end points. On the other hand, if the latter happens on the right boundary, that is, there exists $t_{1}>0$ such that $w\left(x_{1}, t_{1}\right)=0$, where

$$
x_{1}:=\xi_{+}\left(t_{1}\right)=\zeta_{+}^{1}\left(t_{1}+\tau_{1}\right)=\zeta_{+}^{2}\left(t_{1}+\tau_{2}\right) .
$$

Then

$$
\begin{aligned}
& U_{1}\left(x_{1}, t_{1}+\tau_{1}\right)=U_{2}\left(x_{1}, t_{1}+\tau_{2}\right), \\
& U_{1 x}\left(x_{1}, t_{1}+\tau_{1}\right)=U_{2 x}\left(x_{1}, t_{1}+\tau_{2}\right),
\end{aligned}
$$

and so

$$
U_{1 \times x}\left(x_{1}, t_{1}+\tau_{1}\right) \geq U_{2 x x}\left(x_{1}, t_{1}+\tau_{2}\right)
$$

since, otherwise we have $U_{1}\left(x, t_{1}+\tau_{1}\right)<U_{2}\left(x, t_{1}+\tau_{2}\right)$ for $x$ near $x_{1}$ by Taylor's formula. But this contradicts (16).

Using (17), (18), the fact $A_{1}>A_{2}$ and using the equations of $U_{1}$ and $U_{2}$ we have

$$
U_{1 t}\left(x_{1}, t_{1}+\tau_{1}\right)>U_{2 t}\left(x_{1}, t_{1}+\tau_{2}\right) .
$$

Since the normal velocity $V$ in (1) is expressed by

$$
V=\frac{u_{t}}{\sqrt{1+u_{x}^{2}}}
$$

we see that at the point $\left(x_{1}, U_{1}\left(x_{1}, t_{1}+\tau_{1}\right)\right)=\left(x_{1}, U_{2}\left(x_{1}, t_{1}+\tau_{2}\right)\right)$, the normal velocity $V_{1}$ of $U_{1}$ and the normal 
velocity $V_{2}$ of $U_{2}$ satisfies

$$
V_{1}>V_{2} \text {. }
$$

This means that, in a small time-interval around $t=t_{1}$, the graph of $U_{1}$ moves along the boundary $\partial_{+} \Omega$ faster than the graph of $U_{2}$. This, however, contradicts the fact $U_{1}>U_{2}$ and the assumption that $U_{1}\left(x_{1}, t_{1}+\tau_{1}\right)=U_{2}\left(x_{1}, t_{1}+\tau_{2}\right)$. This proves (15).

Now taking $x=x_{0}$ and $t=T_{2}$ in (15) and using the definitions of $T_{i}$ we have

$$
U_{1}\left(x_{0}, T_{2}+\tau_{1}\right)>U_{2}\left(x_{0}, T_{2}+\tau_{2}\right)=U_{2}\left(x_{0}, \tau_{2}\right)+p=U_{1}\left(x_{0}, \tau_{1}\right)+p=U_{1}\left(x_{0}, T_{1}+\tau_{1}\right) .
$$

By the fact $U_{1 t}>0$ in (7) we have $T_{2}>T_{1}$. This implies that $c\left(A_{1}, H, g, p, \alpha_{g}\right)>c\left(A_{2}, H, g, p, \alpha_{g}\right)$ by the definition of $c$ in (6). This proves 1 ) of the Main Theorem.

\subsection{Dependence of $c$ on $H$}

In this subsection we study the dependence of $c$ on $H$ and prove Main Theorem 2). Since only $H$ is varying, for simplicity, in this part we only indicate $H$ but omit all the other parameters in the notations $\Omega, U, c, B, J, K_{i}, \cdots$.

Lemma 1 Assume that $A H>\sin \alpha_{g}$. Then for any $h \in(0, H)$, there holds

$$
U_{x}(h, t ; H)<-\tan \alpha_{g}+2 J(H-h)+\frac{p}{H-h}+p J B .
$$

Proof Let $y_{0} \in(0, p)$ such that $g_{p}^{\prime}\left(y_{0}\right)=\tan \alpha_{g}$. Denote

$$
y_{n}:=y_{0}+n p(n \in \mathbb{Z})
$$

and

$$
x_{0}:=H+g_{p}\left(y_{0}\right)=H+g_{p}\left(y_{n}\right) .
$$

Then there exists $t_{0}$ such that, for $t_{n}:=t_{0}+n T_{p}(n \in \mathbb{Z})$,

$$
U\left(x_{0}, t_{n} ; H\right)=y_{n} \text { and } U_{x}\left(x_{0}, t_{n} ; H\right)=-\tan \alpha_{g} .
$$

Since $U_{t}(x, t ; H)>0$ we have

$$
\frac{U_{x x}}{1+U_{x}^{2}}+A \sqrt{1+U_{x}^{2}}>0
$$

and so

$$
U_{x x}>-A\left(1+U_{x}^{2}\right)^{3 / 2} \geq-A\left(1+S^{2}\right)^{3 / 2}=-J .
$$

For any $h \in(0, H)$, set $P_{n}:=\left(h, U\left(h, t_{n} ; H\right)\right), R_{n}:=\left(H, U\left(H, t_{n} ; H\right)\right)$. Denote the straight line passing $P_{n}$ and $R_{n+1}$ by $\ell_{n}$ and denote its slope by $k_{n}$, then by (20) we have

$$
\begin{aligned}
k_{n} & =\frac{U\left(H, t_{n+1} ; H\right)-U\left(h, t_{n} ; H\right)}{H-h} \\
& =\frac{U\left(H, t_{n+1} ; H\right)-U\left(h, t_{n+1} ; H\right)+p}{H-h} \\
& =U_{x}\left(h+\theta_{1}(H-h), t_{n+1} ; H\right)+\frac{p}{H-h} \\
& =-\tan \alpha_{g}+U_{x x}\left(x_{0}+\theta_{2} \rho, t_{n+1} ; H\right) \rho+\frac{p}{H-h} \\
& \leq-\tan \alpha_{g}-J \rho+\frac{p}{H-h} \\
& <-\tan \alpha_{g}+p J B+J(H-h)+\frac{p}{H-h},
\end{aligned}
$$


where $\rho:=h+\theta_{1}(H-h)-x_{0}$ and $\theta_{1}, \theta_{2} \in[0,1]$. For any $t \in\left[t_{n}, t_{n+1}\right]$, since $U_{t}>0$, the graph of $U(x, t ; H)$ must contact $\ell_{n}$ at some point in $[h, H] \times \mathbb{R}$. If we denote the $x$-coordinate of the contact point by $x(t) \in$ $[h, H]$, then $U_{x}(x(t), t ; H) \leq k_{n}$. Using (20) again we have

$$
\begin{aligned}
U_{x}(h, t ; H) & =U_{x}(x(t), t ; H)+U_{x x}\left(x(t)+\theta_{3}(h-x(t)), t ; H\right)(h-x(t)) \\
& \leq k_{n}+J(H-h)<-\tan \alpha_{g}+2 J(H-h)+\frac{p}{H-h}+p J B,
\end{aligned}
$$

where $\theta_{3} \in[0,1]$. This proves (19).

Lemma 2 Assume that $A H>\sin \alpha_{g}$ and $p<K_{1}(H)$. Then $c(H)<c(H+h)$, where $h=H-d(A, g, p)$.

Proof Since $p<K_{1}(H)$ we have by (12) $d<H$ and $Q<\tan \alpha_{g}$. The definition of $d$ and $h=H-d$ imply that $2(H-h) J=p /(H-h)$ and so

$$
2(H-h) J+\frac{p}{H-h}=2 \sqrt{2 J p} .
$$

So by Lemma 1 we have

$$
\begin{aligned}
U_{x}(h, t ; H) & <-\tan \alpha_{g}+2(H-h) J+\frac{p}{H-h}+p J B \\
& =-\tan \alpha_{g}+2 \sqrt{2 J p}+p J B=-\tan \alpha_{g}+Q<0 .
\end{aligned}
$$

Since $U(x, t ; H)$ is even in $x$ we have $U_{x}(-h, t ; H)>0$. Set $W(x, t):=U(x-h, t ; H)$. Then

$$
\left\{\begin{array}{l}
W_{t}=\frac{W_{x x}}{1+W_{x}^{2}}+A \sqrt{1+W_{x}^{2}}, \quad 0<x<\zeta(t)+h, t \in \mathbb{R}, \\
W_{x}(\zeta(t)+h, t)=-g_{p}^{\prime}(W(\zeta(t)+h, t)), t \in \mathbb{R}, \\
W_{x}(0, t)>0, \quad t \in \mathbb{R} .
\end{array}\right.
$$

On the other hand, replacing $H$ by $H+h$ in the problem (3)-(4), we have a unique periodic traveling wave $U(x, t ; H+h)$ with average speed $c(H+h)$ for this new problem. Using the fact $U_{x}(0, t ; H+h)=0(t \in \mathbb{R})$ and using a similar discussion as in subsection 3.1 we can compare $U(x, t ; H+h)$ with $W(x, t)$ in the domain $\Omega(H+h) \cap\{x>0\}$, and to conclude that $U(x, t ; H+h)$ moves faster than $W(x, t)$. So we obtain $c(H+h)>c(H)$. This proves the lemma.

Proof of Main Theorem 2. Set $H_{k}:=2^{k-1} H-\left(2^{k-1}-1\right) d(k=1,2, \cdots)$ as in the statement of 2), then

$$
0<H=H_{1}<H_{2}<\cdots, \quad \sin \alpha_{g}<A H_{k}
$$

and

$$
p<K_{1}\left(H_{k}\right)(k=1,2, \cdots)
$$

by $p<K_{1}(H)$. Define $h_{k}:=H_{k}-d(k=1,2, \cdots)$, then

$$
0<h_{k}<H_{k}, H_{k}+h_{k}=H_{k+1}(k=1,2, \cdots) \text {. }
$$

Using Lemma 2 to $H=H_{k}$ we have $c\left(H_{k}\right)<c\left(H_{k}+h_{k}\right)=c\left(H_{k+1}\right)$. This proves 2) of the main theorem.

Remark If we take $A=0$ in the original problem, then to guarantee the existence of periodic traveling waves we should modify the boundary conditions a little. For example, one can consider a problem such that the curve $\gamma_{t}$ always has a positive/negative slope on the right/left boundary. In this case, a similar discussion as in [1] shows that the problem has a unique periodic traveling wave $U$ with average speed $c$. Moreover $U_{t}>0$, or equivalently, $U_{x x}>0$. Using these results and using a similar discussion as above we can show that $\partial c / \partial H<0$. In other words, the monotonic dependence of $c$ on $H$ is completely different from the case $A>0$.

\subsection{Dependence of $c$ on $g$ and $\alpha_{g}$}

In this subsection we study the dependence of $c$ on $g$ and $\alpha_{g}$. Similar as above, we only indicate $g$ and $\alpha_{g}$ but 
omit all the other parameters in the notations $U, c, \cdots$ for simplicity.

First we note that a classical traveling wave solution of (3) (with a constant speed and a constant profile) is generally written in the form $u(x, t)=\varphi(x)+c_{0} t$. Substituting this form into (3) yields

$$
c_{0}=\frac{\varphi_{x x}}{1+\varphi_{x}^{2}}+A \sqrt{1+\varphi_{x}^{2}} .
$$

In addition, considering the normalization and the symmetry of $\Omega$, we impose the following initial condition:

$$
\varphi(0)=0, \varphi_{x}(0)=0 \text {. }
$$

Denote the solution of (22)-(23) by $\varphi\left(x ; c_{0}\right)$.

Lemma 3 (Lemma 5.1 in) Assume that $A h>\sin \tilde{\alpha}$. Then the constant $\bar{c}=\bar{c}(A, h, \tilde{\alpha})$ defined by

$$
h=\int_{0}^{\tilde{\alpha}} \frac{\cos r d r}{A-\bar{c} \cos r}
$$

satisfies

$$
0<\bar{c}<A, \quad \frac{\partial \bar{c}}{\partial h}>0 .
$$

The solution $\varphi(x ; \bar{c})$ of (22)-(23) satisfies $\varphi_{x}(h ; \bar{c})=-\tan \tilde{\alpha}$.

Lemma 4 Let $\tilde{\alpha} \in\left(0, \frac{\pi}{4}\right), \tilde{B}>0,0<\tilde{S}<1$ and $\tilde{g} \in \mathcal{G}(\tilde{\alpha}, \tilde{B}, \tilde{S})$. Assume that $A H>\sin \tilde{\alpha}$. Then

$$
\bar{c}(A, H, \tilde{\alpha})<c(\tilde{g}, \tilde{\alpha}),
$$

where $c(\tilde{g}, \tilde{\alpha})$ is the average speed of the periodic traveling wave $U(x, t ; \tilde{g}, \tilde{\alpha})$ of (3)-(4) in band domain $\Omega(H, \tilde{g}, p)$.

Proof From (7) we see that $U(x, t ; \tilde{g}, \tilde{\alpha})$ satisfies

$$
U_{x}(0, t ; \tilde{g}, \tilde{\alpha})=0, \quad U_{x}(H, t ; \tilde{g}, \tilde{\alpha}) \geq-\tan \tilde{\alpha}
$$

for all $t \in \mathbb{R}$. So $U$ is an upper solution of

$$
\left\{\begin{array}{l}
u_{t}=\frac{u_{x x}}{1+u_{x}^{2}}+A \sqrt{1+u_{x}^{2}}, \quad x \in(0, H), \\
u_{x}(0, t)=0, u_{x}(H, t)=-\tan \tilde{\alpha}
\end{array}\right.
$$

On the other hand, by Lemma $3 \bar{u}(x, t):=\varphi(x ; \bar{c}(A, H, \tilde{\alpha}))+\bar{c}(A, H, \tilde{\alpha}) t$ is a classical traveling wave of (27). So we can use comparison principle as in subsection 3.1 for $\bar{u}(x, t)$ and $U(x, t ; \tilde{g}, \tilde{\alpha})$ on the interval $x \in[0, H]$ to conclude that $c(\tilde{g}, \tilde{\alpha})>\bar{c}(A, H, \tilde{\alpha})$.

Proof of Main Theorem 3. We write $g$ and $\alpha_{g}$ as $g_{0}$ and $\alpha_{0}$, respectively. For any $k=0,1,2, \cdots, N-1$, since

$$
p<K_{2}\left(g_{0}, \alpha_{0}\right), B\left(g_{k}\right) \leq B\left(g_{0}\right), S\left(g_{k}\right) \leq S\left(g_{0}\right)<1,
$$

we have

$$
\begin{gathered}
A<J\left(g_{k}\right) \leq J\left(g_{0}\right), Q\left(g_{k}\right) \leq Q\left(g_{0}\right), \\
d\left(g_{k}\right)=\sqrt{\frac{p}{2 J\left(g_{k}\right)}}<\sqrt{\frac{p}{2 A}}<H, h_{k}:=H-d\left(g_{k}\right)>0, \\
2\left(H-h_{k}\right) J\left(g_{k}\right)=\frac{p}{H-h_{k}}\left(\text { by the definition of } d\left(g_{k}\right)\right),
\end{gathered}
$$

and

$$
\tan \alpha_{k+1}=\tan \alpha_{0}-(k+1) Q\left(g_{0}\right)=\tan \alpha_{k}-Q\left(g_{0}\right)>0
$$


By Lemma 1 and the definitions of $d\left(g_{k}\right)$ and $Q\left(g_{k}\right)$ we have

$$
\begin{aligned}
U_{x}\left(h_{k}, t ; g_{k}, \alpha_{k}\right) & <-\tan \alpha_{k}+2\left(H-h_{k}\right) J\left(g_{k}\right)+\frac{p}{H-h_{k}}+J\left(g_{k}\right) B\left(g_{k}\right) p \\
& =-\tan \alpha_{k}+2 \sqrt{2 J\left(g_{k}\right) p}+J\left(g_{k}\right) B\left(g_{k}\right) p \\
& \leq-\tan \alpha_{k}+Q\left(g_{0}\right)=-\tan \alpha_{k+1}<0 .
\end{aligned}
$$

So $U\left(x, t ; g_{k}, \alpha_{k}\right)$ is a lower solution of

$$
\left\{\begin{array}{l}
u_{t}=\frac{u_{x x}}{1+u_{x}^{2}}+A \sqrt{1+u_{x}^{2}}, \quad x \in\left(0, h_{k}\right), \\
u_{x}(0, t)=0, u_{x}\left(h_{k}, t\right)=-\tan \alpha_{k+1} .
\end{array}\right.
$$

Replacing $h, \tilde{\alpha}$ in Lemma 3 by $h_{k}, \alpha_{k+1}$, respectively, we know that

$$
\bar{w}(x, t):=\varphi\left(x ; \bar{c}\left(A, h_{k}, \alpha_{k+1}\right)\right)+\bar{c}\left(A, h_{k}, \alpha_{k+1}\right) t
$$

is a classical traveling wave of (28). So we can use comparison principle for $\bar{w}$ and $U\left(x, t ; g_{k}, \alpha_{k}\right)$ in the interval $x \in\left[0, h_{k}\right]$ to conclude that

$$
c\left(g_{k}, \alpha_{k}\right)<\bar{c}\left(A, h_{k}, \alpha_{k+1}\right) .
$$

On the other hand, replacing $(\tilde{g}, \tilde{\alpha})$ by $\left(g_{k+1}, \alpha_{k+1}\right)$ in Lemma 4 we have

$$
c\left(g_{k+1}, \alpha_{k+1}\right)>\bar{c}\left(A, H, \alpha_{k+1}\right) \text {. }
$$

Combining the above inequalities with (25) we have

$$
c\left(g_{k+1}, \alpha_{k+1}\right)>\bar{c}\left(A, H, \alpha_{k+1}\right)>\bar{c}\left(A, h_{k}, \alpha_{k+1}\right)>c\left(g_{k}, \alpha_{k}\right) .
$$

This proves Main Theorem 3).

\section{Some Numerical Simulation Results}

In this section we present some numerical simulation figures. Figure 2 indicates that the average speed $c$ is strictly increasing in the basic width $H$ of the domain.

Figure 3 indicates that the average speed $c$ is strictly decreasing in the maximum opening angle $\alpha$.

Figure 4 indicates that the average speed $c$ is strictly increasing in the period $p$ of $g$.

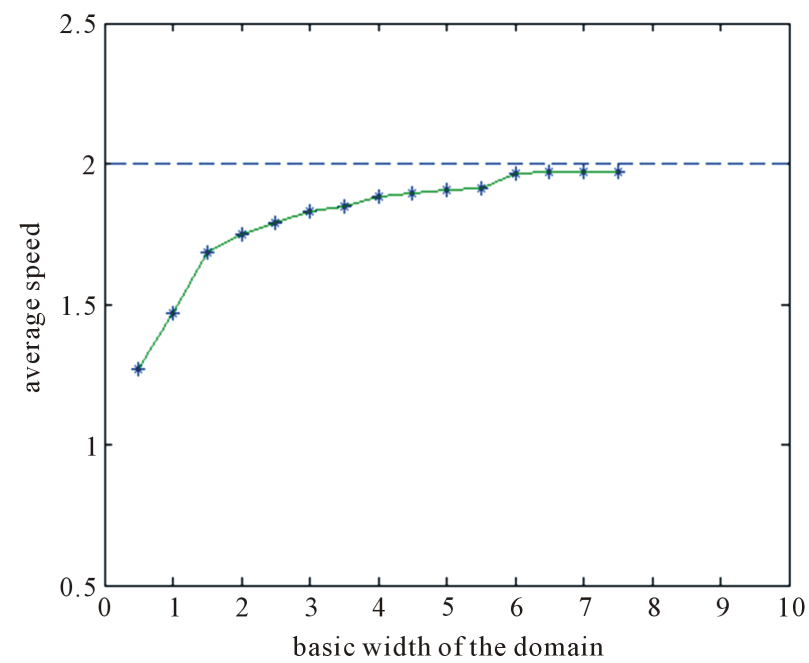

Figure 2. The monotonic dependence of $c$ on $H$. 


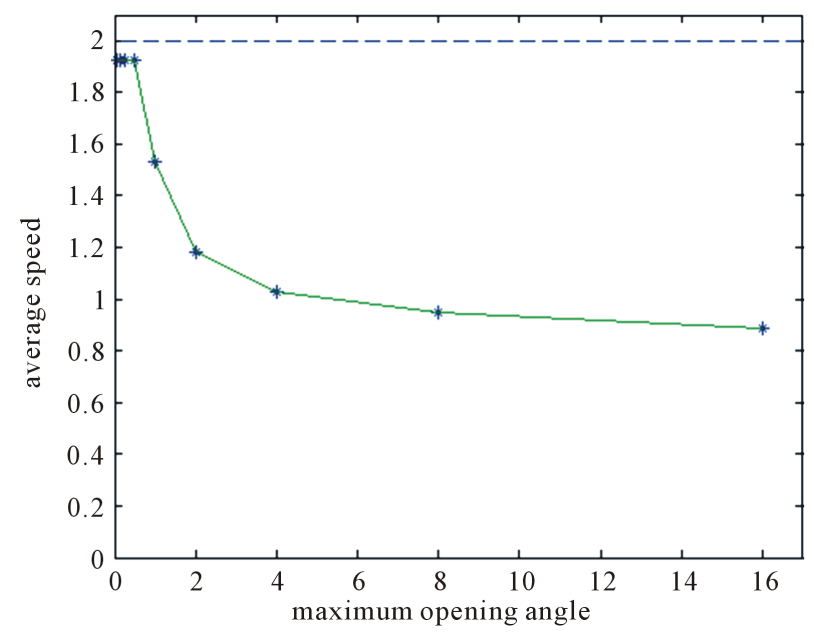

Figure 3. The monotonic dependence of $c$ on $\alpha$.

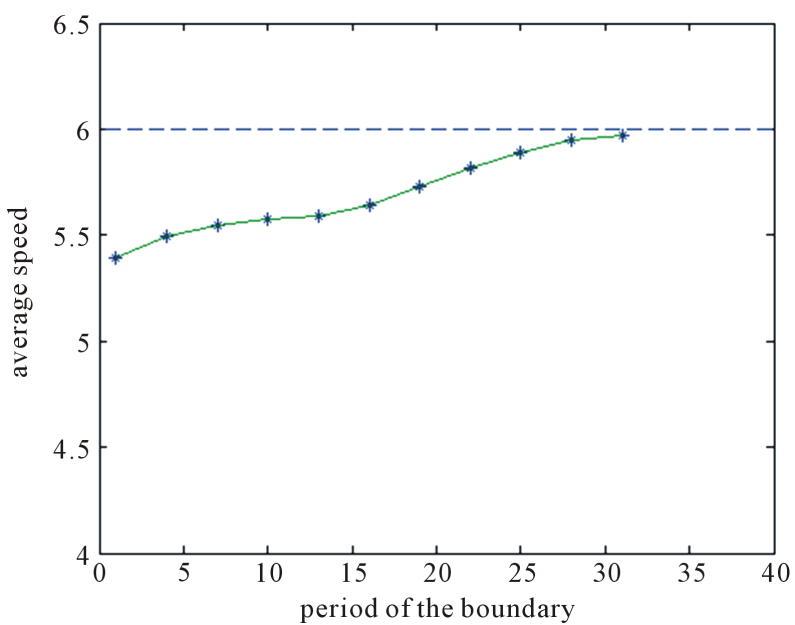

Figure 4. The monotonic dependence of $c$ on $p$.

The results shown in Figure 2 and Figure 3 are partially proved in the main theorem. The dependence of $c$ on $p$ is very difficult, and we have no analytic result so far.

\section{References}

[1] Matano, H., Nakamura, K.I. and Lou, B. (2006) Periodic Traveling Waves in a Two-Dimensional Cylinder with SawToothed Boundary and Their Homogenization Limit. Networks and Heterogeneous Media, 1, 537-568. http://dx.doi.org/10.3934/nhm.2006.1.537

[2] Alfaro, M., Hilhorst, D. and Matano, H. (2008) The Singular Limit of the Allen-Cahn Equation and the FitzHugh-Nagumo System. Journal of Differential Equations, 245, 505-565. http://dx.doi.org/10.1016/j.jde.2008.01.014

[3] Lou, B. (2007) Singular Limits of Spatially Inhomogeneous Convection-Reaction-Diffusion Equation. Journal of Statistical Physics, 129, 509-516. http://dx.doi.org/10.1007/s10955-007-9400-3

[4] Nakamura, K.I., Matano, H., Hilhorst, D. and Schatzle, R. (1999) Singular Limits of Spatially Inhomogeneous Convection-Reaction-Diffusion Equation. Journal of Statistical Physics, 95, 1165-1185. http://dx.doi.org/10.1023/A:1004518904533

[5] Cioranescu, D. and Donato, P. (1999) An Introduction to Homogenization. Oxford University Press, Oxford.

[6] Cioranescu, D. and Saint Jean Paulin, J. (1999) Homogenization of Reticulated Structures. Springer-Verlag, New York.

[7] Protter, M.H. and Weinberger, H.F. (1967) Maximum Principles in Differential Equations. Prentice Hall, Englewood Cliffs, 172-173. 
Scientific Research Publishing (SCIRP) is one of the largest Open Access journal publishers. It is currently publishing more than 200 open access, online, peer-reviewed journals covering a wide range of academic disciplines. SCIRP serves the worldwide academic communities and contributes to the progress and application of science with its publication.

Other selected journals from SCIRP are listed as below. Submit your manuscript to us via either submit@scirp.org or Online Submission Portal.
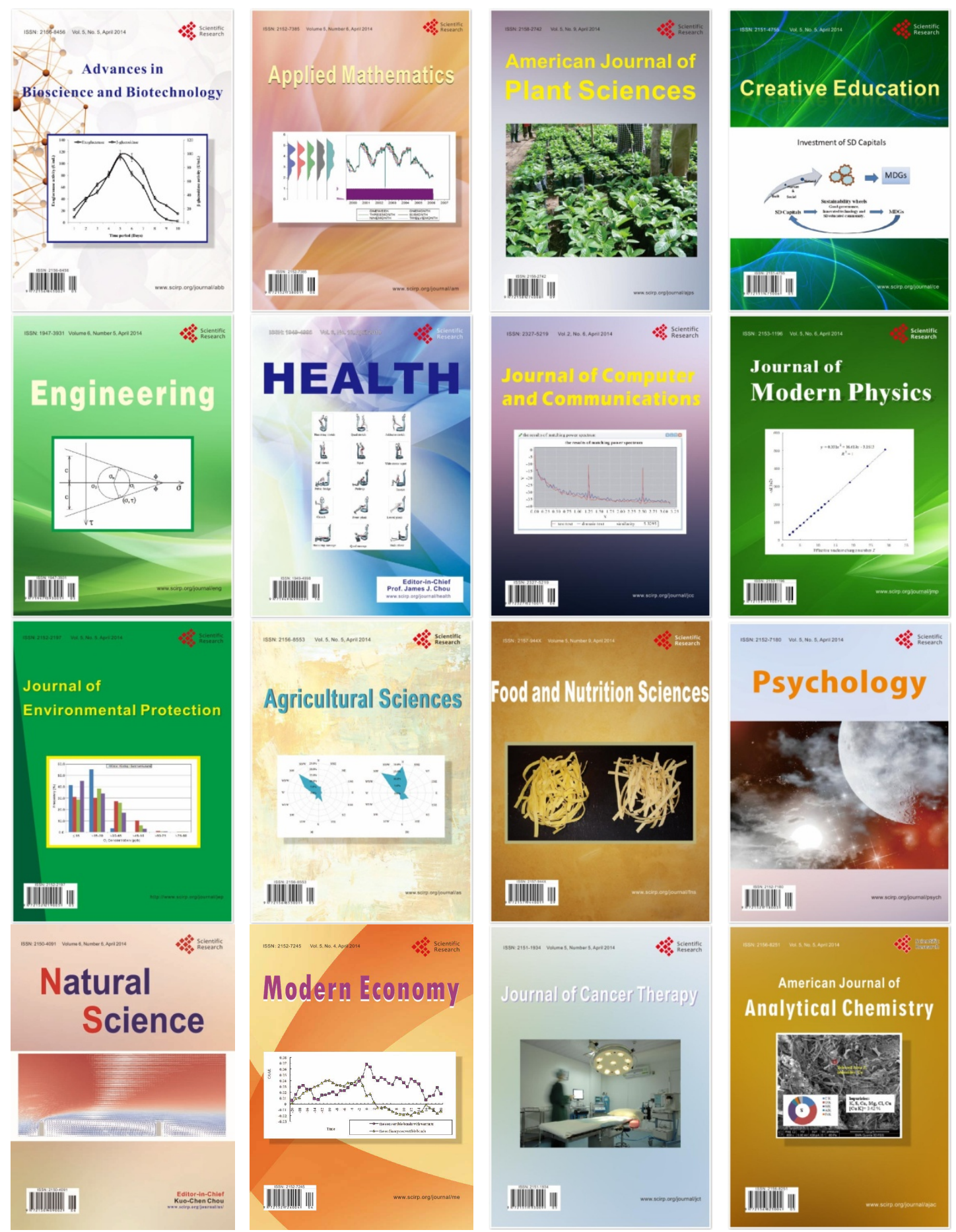